\title{
Acquisition as a function of $S-R$ frequency of occurrence under ORM, OSM, or S-R associative conditions'
}

BARBARA WATTS AND JAMES F. VOSS UNIVERSITY OF PITTSBURGH

\begin{abstract}
Abstraet
Paired-associate experiments which have involved deletion of the response (\%ORM) or the stimulus (\%OSM) on a proportion of the trials confound the effects of the respective response or stimulus occurrence with frequency of S-R occurrence. The present experiment was designed to separate the effects attributable to response (or stimulus) occurrence per se and S-R frequency of occurrence. The experimental conditions consisted of paired-associates presented for eight different frequencies of occurrence under ORM, OSM, and the usual S-R associative procedure. It was found that $\mathrm{S}-\mathrm{R}$ frequency only is significant and that response or stimulus occurrence per se is not important.
\end{abstract} Introduction

Experiments in which responses of paired-associates were deleted on a proportion of trial (\%ORM) generally have indicated that performance decreases as percent response occurrence decreases, e.g., Goss, Morgan, \& Golin (1959). Similarly, stimulus deletion on a proportion of paired-associate trials (\%OSM) also yielded inferior performance (Schulz, 1963).

The \%ORM and \%OSM paradigms, however, involve a methodological confounding. Specifically, the \%ORM conditions confound frequency of response with frequency of S-R pairing. Thus, a significant difference in performance between the $100 \%$ and $50 \%$ ORM conditions may be attributed to a difference of response frequency and/or S-R frequency. Similarly, a significant difference between $100 \%$ and 50\% OSM conditions may be attributed to a difference in frequency of stimulus occurrence and/or a difference of S-R occurrence. The purpose of the present experiment was to provide information regarding whether response (or stimulus) frequency and/or S-R frequency is (are) the important variable(s) in the \%ORM and \%OSM studies.

\section{Procedure}

Eight paired-associates were presented for 80 trials by the anticipation method with the modification that one paired-associate occurred only on 10 of the trials, one paired-associate occurred on 20 trials, ...., and one paired-associate occurred on all 80 trials. Thus, frequency of occurrence of S-R was varied from 10 to 80 presentations in steps of 10 . The particular trials on which the paired-associates occurred were assigned randomly within two 40-trial blocks. The pairedassociates were presented via film-strip projector at a $2: 2$ rate with a $4-\mathrm{sec}$. intertrial interval. The order of the eight paired-associates on each trial was random.

Six experimental conditions were employed in which the stimuli and S-R pairings of the eight pairedassociates were presented identically and materials on the non paired-associate trials were varied. Condition A: on trials when the paired-associates were not presented, two blank frames occurred. Condition $\mathrm{A}_{\mathrm{c}}$ : identical to Condition A except that instead of two blank frames, two geometric forms were presented respectively (Schulz, 1963). The Ss said the name of the geometric forms upon presentation. The geometric form condition was employed as a rehearsal control. Condition OSM: identical to Condition A except that for non pairedassociate trials, the first $2 \mathrm{sec}$. consisted of a blank and the second $2 \mathrm{sec}$. of the response item of the particular paired-associate. Condition $\mathrm{OSM}_{\mathrm{C}}$ : identical to Condition OSM except that the control geometric form procedure was used in place of the blank. Condition ORM: identical to Condition A except that on non pairedassociate trials, the stimulus of the paired-associate was presented for $2 \mathrm{sec}$. followed by a 2-sec. blank. Condition $\mathrm{ORM}_{\mathrm{c}}$ : identical to Condition ORM except that the control geometric form procedure was used in place of the blank. It should be noted that the OSM and ORM conditions represent the OSM and ORM conditions of previous experimentation and that variation in S-R frequency of the eight paired-associates under the OSM and ORM conditions represents $12.5 \%$ $25.0 \%, 37.5 \%, 50.0 \%, 62.5 \%, 75.0 \%, 87.5 \%$, and $100 \%$ OSM or ORM conditions, respectively.

A control for S's responding was employed since the only legitimate way to compare performance under the six experimental conditions was to consider correct responses on the paired-as sociate trials only. Thus, in order to restrict the trials on which $\mathrm{S}$ anticipated, a horizontal line was used as an indicator that $\mathrm{S}$ should anticipate. Otherwise, a vertical line was used to indicate that $\mathrm{S}$ should not try to anticipate. Thus, for the S-R frequency of 10 , there were 10 possible correct responses for all six experimental conditions; for the S-R frequency of 20 there were 20 possible correct responses, etc.

\section{Materials}

Low frequency words of Horton \& Kjeldergaard (1960) were assigned to the eight paired-associate frequency conditions by use of a table of random numbers. Two such randomizations were employed which were orthogonal to the experimental conditions. 


\section{Subjects}

The Ss were 96 University of Pittsburgh Introductory Psychology students. Sixteen Ss were assigned randomly to each of the experimental conditions. The instructions were those typically used in paired-associate experiments, with the inclusion of a double articulation procedure.

\section{Results}

Since the frequency conditions provided differential opportunity for correct responses, percent correct responses was employed. Percent correct responses increased in a negatively accelerated manner over the S-R frequency conditions. ${ }^{2}$ The percent correct response data were submitted to an arcsin transformation and an analysis of variance revealed that the $S-R$ frequency source of variation is significant, $F(7,630)=$ $55.56 ; \mathrm{p}<.01$. The analysis of variance also revealed that the experimental condition source of variation is not significant, $F(5,90)<1.00$. Analysis also revealed that the difference between the non-geometric conditions and geometric form control conditions is not significant, $\mathrm{F}(1,94)=2.50$ 。

\section{Diseussion}

The major implication of the present findings for \%ORM and \%OSM investigations is that S-R frequency yields a significant performance effect and that deletion of the response (\%ORM) or deletion of the stimulus (\%OSM) apparently has little effect upon performance. Previous \%ORM and \%OSM results thus may be attributed to differences of S-R frequency and not to differences in $\mathbf{R}$ (\%ORM), or S (\%OSM) occurrence.

The present experiment, employing low frequency words, consists of a relatively small degree of response learning (Underwood \& Schulz, 1960)。With materials requiring more response learning, however, acquisition is likely facilitated when the response is presented (OSM). Similarly, the use of geometric forms as a control is likely more effective when response learning is necessary (Schulz, 1963). The present finding thus may be interpreted to relate to the associative phase of acquisition, and in particular, to indicate that frequency of S-R occurrence contributes to associative learning, but stimulus or response occurrence per se does not so contribute. These findings are in agreement with Camp (1960), who presented stimuli and responses individually or as S-R pairs and found that correct association performance was related only to frequency of $S-R$ occurrence.

\section{References}

Camp, B. W. "Association" of nonsense syllables following varied learning conditions. J. exp. Psychol., 1960, 59, 35-40.

Goss, A. E., Morgan, C. H., \& Golin, S. J. Paired-associates learning as a function of percentage of occurrence of response members (reinforcement). J. exp. Psychol., 1959, 57, 96-104.

Horton, D. L., \& Kjeldergaard, P. M. An experimental analysis of associative factors in mediated generalizations. Psychol. Monogr., 1961, 75, No. 11.

Schulz, R. W. Personal communication, 1963

Underwood, B. J., \& Schulz, R. W. Meaningfulness and verbal learning. New York: Lippincott, 1960.

\section{Notes}

1. The experiment reported in this paper was supported by the National Institute of Child Growth and Human Development (HD00957-02).

2. The percent correct responses for the eight frequency conditions was as follows: 10-67.3; 20-74.3; 30-73.7; 40-80.4; 50-78.5; 60-86.9: 70-91.2: 80-86.6. 